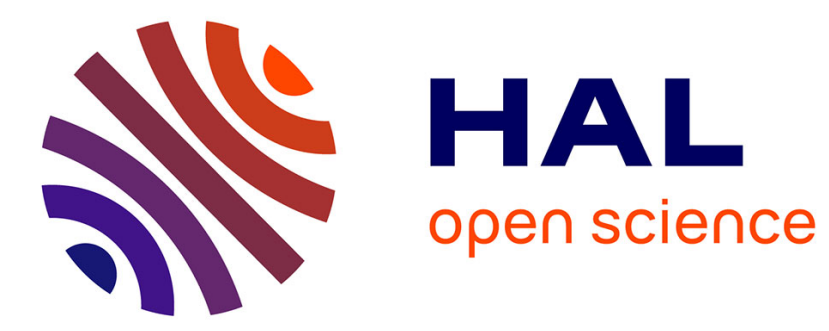

\title{
Background on Fracture Mechanics
}

\author{
Huy Duong Bui, J.B. Leblond, N. Stalin-Muller
}

\section{To cite this version:}

Huy Duong Bui, J.B. Leblond, N. Stalin-Muller. Background on Fracture Mechanics. Handbook of Materials Behavior Models 2, Academic Press, pp.549-565, 2001, 10.1016/B978-012443341-0/50061-

2. hal-00112289

\section{HAL Id: hal-00112289 \\ https://hal.science/hal-00112289}

Submitted on 18 Feb 2020

HAL is a multi-disciplinary open access archive for the deposit and dissemination of scientific research documents, whether they are published or not. The documents may come from teaching and research institutions in France or abroad, or from public or private research centers.
L'archive ouverte pluridisciplinaire HAL, est destinée au dépôt et à la diffusion de documents scientifiques de niveau recherche, publiés ou non, émanant des établissements d'enseignement et de recherche français ou étrangers, des laboratoires publics ou privés. 


\section{Background on Fracture Mechanics}

Huy Duong Bui ${ }^{1,2}$, J-B. Leblond ${ }^{3}$, N. Stalin-Muller ${ }^{1}$

${ }^{1}$ Laboratoire de Mécanique des Solides, Ecole Polytechnique, 91128 Palaiseau, France

${ }^{2}$ Electricité de France, RED, Clamart, France

${ }^{3}$ Laboratoire de Modélisation en Mécanique, Université de Pierre et Marie Curie, 8 rue du Capitaine Scott, 75015 Paris, France

\subsubsection{VALIDITY}

Linear elastic fracture mechanics (LEFM) is based on the analysis of cracks in linear elastic materials. It provides a tool for solving most practical problems in engineering mechanics, such as safety and life expectancy estimation of cracked structures and components. The main success of the theory is based precisely upon linearity, which makes it possible to combine very simply 
the theoretical, numerical, and experimental analyses of fracture. Today, stress analyses of the complex geometry of structures as well as of test specimens are provided by powerful computers using finite element methods. The computed stress-intensity factor $K_{\mathrm{I}}$ in mode I, which governs the strength of the singular field near the crack tip, depends linearly on the applied load. Its critical value, related to the toughness $K_{\mathrm{lc}}$ of the material, is obtained by measuring the critical load $F_{c}$ at the onset of unstable crack propagation. Strictly speaking, this scheme is an ideal one and can be used only under restricted conditions involving the geometry of the specimen, the flow stress level, etc. For example, there exist empirical conditions on the crack length $a$ which must be greater than $b$, the specimen thickness, and the flow stress $a \gg b \gg 2.5\left(K_{\mathrm{Ic}} / \sigma_{O}\right)^{2}$. These empirical conditions result from test data and correspond to the smallness of the process zone size compared to a length scale. It also expresses the condition of smallness of plastic deformation when compared to elastic strain. This is the small-scale yielding assumption.

\subsubsection{STRESS OR VELOCITY FIELDS SINGULARITIES?}

Without experiments, it is not easy to know to what extent a theory may be "good" enough for practical use. Nor is it simple to do "rigorously" experimental work to validate the theory. The rigor consists of carefully checking the actual conditions of the experiments. For example, the toughness of material is determined by testing specimens subjected to monotonous increasing loads only when some onset of crack propagation is observed. In an elastic medium with a propagating crack with the velocity $V$, in plane strain or in antiplane strain loading, it is well known that the singular stress fields near the crack tip $(r \rightarrow 0)$ are governed by the stress-intensity factors in modes $j=\mathrm{I}$, II, III:

$$
K_{j}=\lim (r \rightarrow 0) \sigma_{2 i(j)}(2 \pi r)^{1 / 2} \quad i(\mathrm{I})=2, i(\mathrm{II})=1, i(\mathrm{III})=3
$$

The velocity field $d u_{i} / d t$ is also singular at the crack tip and yields an alternative mean for characterizing the strength of the singularity. As a matter of fact, near the crack tip, the velocity is related to the displacement by $d u_{i} / d t \cong-V \partial u_{i} / \partial x_{1}$, or to the crack opening displacement (COD) $\Phi_{i}$, because in the vicinity of the crack tip one has $\Phi_{i} \equiv\left[u_{i}\left(x_{1}-V t, x_{2}\right)\right]$, where [.] denotes the jump across the crack surface. Hence there exist three crack opening displacement intensity factors, in the form

$$
K_{j}^{(u)}=\lim (r \rightarrow 0) \gamma \Phi_{i(j)}(2 \pi / r)^{1 / 2}, \quad i(\mathrm{I})=2, i(\mathrm{II})=1, i(\mathrm{III})=3
$$




\subsubsection{PLANE STRAIN, ANTIPLANE STRAIN, AND PLANE STRESS}

Equation 2 is valid for plane strain modes I and II, with $\gamma=\mu /(k+1)$, and $k=3-4 v, \mu$ being the shear modulus, and $v$ the Poissons ratio. The mode III fracture corresponds to the antiplane shear loading with the value $\gamma=\mu / 4$.

We do not give here the usual value $\gamma$ for the plane stress case, because contrary to common belief, the formula for plane stress is questionable. To show that, remember that the plane stress assumption implies that the normal strain $\varepsilon_{33}\left(x_{1}, x_{2}\right)=-v\left(\sigma_{11}+\sigma_{22}\right) / E$ is a function of the first two coordinates $x_{1}, x_{2}$ because $\sigma_{i j}$ does. The compatibility equation for $\varepsilon_{33}\left(x_{1}, x_{2}\right)$ implies that all partial second derivatives with respect to $x_{1}, x_{2}$ are equal to zero; hence it may be a linear function of the coordinates $\varepsilon_{33}\left(x_{1}, x_{2}\right)=a x_{1}+b x_{2}+c$. Clearly, the compatibility condition is violated in the vicinity of the crack tip because precisely the strain component $\varepsilon_{33}(r, \theta)$ as given in the textbook is singular as $r^{-1 / 2}$. This contradicts the linear function variation. Hence the common belief that for thin shells the formula for plane stress prevails near the crack tip is questionable.

\subsubsection{DYNAMIC STRESS INTENSITY FACTORS}

In quasi-static linear elasticity, both definitions, either by the stress (Eq. 1) or by the COD (Eq. 2), are strictly equivalent. However, in dynamic crack propagation, when inertial forces cannot be neglected, Eqs. 1 and 2 lead to two distinct crack tip parameters. It has been proved that there are universal relationships between these parameters:

$$
K_{j}^{(u)}=f_{j}(V) K_{j}
$$

with for mode $j=$ I, II, III

$$
\begin{aligned}
f_{\mathrm{I}}(V) & =\left\{4 \beta_{1}\left(1-\beta_{2}^{2}\right)\right\} /(k+1)\left\{4 \beta_{1} \beta_{2}-\left(1-\beta_{2}^{2}\right)^{2}\right\} \\
f_{\mathrm{II}}(V) & =\left\{4 \beta_{2}\left(1-\beta_{2}^{2}\right)\right\} /(k+1)\left\{4 \beta_{1} \beta_{2}-\left(1-\beta_{2}^{2}\right)^{2}\right\} \\
f_{\mathrm{III}}(V) & =1 / \beta_{2}
\end{aligned}
$$

where $\beta_{j}=\left(1-V^{2} / c_{\mathrm{j}}^{2}\right)^{1 / 2}$, with $c_{1}$ the velocity of the compressive P-wave, $c_{2}$ the velocity of the shear $S$-wave, and $k=3-4 v[1]$. The ratio $f_{1}$ is equal to 1 for $V=0$, and greater than 1 for $V \neq 0$, tending to infinity when $V$ approaches the Rayleigh velocity. 
The multiplicity of crack tip parameters raises the question, What is the true parameter measured in testing materials in dynamic fracture, stress- or velocity-intensity factors? If the onset crack propagation velocity $V$ is not negligible, or undetermined, the critical state determining the toughness of the material from experimental results may be differently interpreted by inappropriate formulae for stress-intensity factors.

\subsubsection{SMALL-SCALE YIELDING ASSUMPTION}

This assumption is essential in LEFM theory. It explains the apparent paradoxical statement that a linear elastic theory is capable of describing complex situations involving nonlinearity such as plasticity, viscoplasticity, damage, etc., which prevail in the process zone near the crack tip. Modern asymptotic analyses using some small parameter - for example, the ratio of the process zone size to the crack length - provide a mathematical justification of this well-known concept that everyone in engineering science is familiar with, without any mathematical tool. A good review paper on this topic is provided recently by Willis [17]. We shall consider some aforementioned nonlinearities.

\subsubsection{PLASTICITY AND DAMAGE}

A plastic correction to the linear elastic solution was first given heuristically by Irwin. In the plastic zone, defined by the overstressed region, the normal stress $\sigma_{22}$ is cut off to the constant yield stress $\sigma_{0}$. Later, an exact solution taking account of perfect plasticity was provided by Rice [15] for the antiplane shear loading of a semi-infinite crack. The plastic zone is a circle passing through the crack tip. According to modern terminology, the plastic field is the inner solution while the elastic field governed by the stress asymptotics $\sigma_{3 \mathrm{i}} \cong K_{\mathrm{III}} r^{-1 / 2} g(\theta)$ at infinity, $r \rightarrow \infty$ (not for $r \rightarrow 0$ ), is the outer solution. Rice [15] gives an exact solution matching inner and outer solutions. This solution has been generalized by Bui and Ehrlacher [3] to a notch with the slit width $2 h \neq 0$ and the notch geometry to be determined in such a way that $\sigma_{3 \tau}=\sigma_{0}$ along the notch. It is found that the notch is a cusped cycloid and that the elastic-plastic boundary is a curled cycloid. For the limiting case where $\sigma_{0} \rightarrow \infty$, one recovers Rice's solution for the crack $h \rightarrow 0$ with the circular plastic zone. The notch solution can be interpreted as an elastic-brittle damage model where the material inside the slit has been totally damaged (zero stress state). The slit of thichness $2 h$ is the wake damage zone, and the cusped cycloid is the damage front. 


\subsubsection{VISCOPLASTIC SOLUTION}

The process zone is likely a high-stress region where viscous plastic flow may occur. An asymptotic inner solution for $r \rightarrow 0$ has been provided by Hui and Riedel [10] in the case of steady state propagation of a crack, with the velocity $V$ and without inertial force. The Norton and Hoff visco-elastoplastic law without threshold is considered in the form

$$
\varepsilon^{r}=C_{1} \sigma^{r}+B|\sigma|^{m-1} s \quad(m \geq 3)
$$

(the superscript ${ }^{r}$ means "rate", $\varepsilon^{r}$ is the strain rate, $\sigma^{r}$ is the stress rate, $s$ is the stress deviator, and $|\sigma|$ is the von Mises deviator norm). In antiplane shear loading, the nonlinear equilibrium equation corresponding to this law has been given by Kachanov [11] in terms of the stress function $\psi\left(x_{1}, x_{2}\right)$ :

$$
-V \Delta\left(\partial \psi / \partial x_{1}\right)+\eta \operatorname{div}\left\{|\operatorname{grad} \psi|^{m-1} \operatorname{grad} \psi\right\}=0
$$

$\left(\sigma_{31}=-\psi_{.2}, \sigma_{32}=\psi_{.1}\right)$, with suitable boundary conditions on the crack tip. Readers interested in the numerical solution of Kachanov's equation can refer to Hui and Riedel [10]. We do not go into the details of their numerical solution, but consider qualitatively instead its essential behavior.

The main feature of the solution is that there is an inner solution $\psi(r, \theta, V)$ having the asymptotics $\psi \cong C(V) r^{(m-2) /(m-1)} g(\theta)$ for $r \rightarrow 0$. Not only is the angular function $g(\theta)$ uniquely determined, but the amplitude $C(V)$ as well. There is no free parameter like the stress-intensity factor $K$ or Rice's $J$-integral. We are faced with what is known in nonlinear physics as the soliton solution of a nonlinear wave, like the well-known Korteweg and de Vries soliton in fluid dynamics. The term soliton has the meaning of a solitary wave. The term viscoplastic soliton was coined for the first time in Bui [5]. Let us draw the consequence of an existing match between the inner soliton and the outer elastic solution $\sigma_{3 \mathrm{i}} \cong K_{\mathrm{III}} r^{-1 / 2}$ at infinity $r \rightarrow \infty$. A matching of solution, which can be achieved only by numerical methods, means that there exists a relationship between $K_{\mathrm{III}}$ and the amplitude $C(V)$. This theoretical analysis provides an explanation for the dependence of the toughness on crack velocity, likely due to a viscous phenomenon occurring in the process zone.

\subsubsection{ENERGY CONSIDERATIONS}

Paralleling with the simple approach of LEFM, a more elaborate avenue based on energetic considerations was followed by various authors, and notably by Irwin in the late 1950s and 1960s. This other approach was initiated by Griffith in the 1920s. Griffith showed that an energetic analysis of a quasistatically growing crack led in a natural way to some propagation criterion 
involving a critical value of the so-called energy release rate $G$, which could be related to the derivative of the overall stiffness $K$ (or compliance $C$ ) of the body considered with respect to the crack length $a, G=-(1 / 2) u^{2} d K / d a$ (or $\left.G=(1 / 2) T^{2} \mathrm{~d} C / d a\right)$. This led to a simple interpretation of $G$ in the overall strain-overall stress diagram. From there, Irwin proved a famous relation connecting $G$ to the stress-intensity factors, for example, in plane strain mixed modes I and II

$$
G=\left(1-v^{2}\right)\left(K_{\mathrm{I}}^{2}+K_{\mathrm{II}}^{2}\right) / E
$$

This established the equivalence of Irwin's propagation criterion involving the stress-intensity factor(s) and Grifftith's one involving the energy release rate.

Also, Rice [15] and Cherepanov [6] simultaneously established the existence of a line integral noted $J$ (or $\Gamma$ in Russian), the contour of which must surround the crack tip and which possessed the following nice properties: first, it is independent of the integration contour; second, it is equal to $G$ and thus, by Irwin's formula, connected to the stress-intensity factor(s). If the crack is not loaded in pure mode I, the expression of $J$ involves all three stress-intensity factors, so that not all values of these three quantities can be deduced from that of $J$. However, at least in mixed mode I + II, the problem of separating the modes, that is, of deducing the values of both stressintensity factors from Rice-type integrals, was solved by Bui [4]. The main interest of Rice's J-integral is essentially numerical: indeed, it allows us to evaluate the stress-intensity factors through numerical integration on a path located far from the crack tip, thus circumventing the difficulty of such an evaluation from the unavoidably somewhat inaccurate values of the near-tip mechanical fields.

\subsubsection{DUCTILE FRACTURE}

The first approach to ductile fracture, that is, fracture of materials undergoing considerable plastic deformation prior to failure, was based on Rice's $J$-integral. Indeed, the property of invariance of $J$ with respect to the integration path remains true in nonlinear elasticity, which made it tempting to apply it to problems involving plasticity (unloading effects being disregarded). In this approach, propagation was assumed to occur when some critical value of $J$ was reached, this critical value being allowed to depend upon the crack length. This theory was later named the global approach to ductile rupture, since it did not rely on any detailed micromechanical analysis of the mechanism of ductile rupture, that is, nucleation, growth, and finally coalescence of voids, through breaking of the 
inclusions-matrix interfaces or the inclusions themselves and subsequent plastic flow of the matrix. In contrast, the more modern theory of ductile rupture, named the local approach, is based on such an analysis. As years passed, the superiority of the latter approach has become clear, although the older one is still widely used in practical problems. A first, a seminal contribution was made by Rice and Tracey [16]; it consisted of an approximate analysis of the growth of a void in an inifinite plastic matrix loaded arbitrarily at infinity. Later, approximate criteria for porous plastic solids were proposed by Rousselier [14], who extended Rice and Tracey's void model, and Gurson [9], who proposed a model based on an approximate limit analysis of a typical elementary cell in a porous medium (hollow sphere). This model has become very popular. It was recently extended by Gologanu et al. [8] to incoporate void shape effects, which were neglected in Gurson's model of spherical voids. Void growth in plastic solids is thus now fairly understood and described by suitable models. Therefore, the theoretical analysis of coalescence has now become the major challenge in the local approach to ductile rupture.

This phenomenon is very complex and is influenced by numerous factors, such as void shape, inhomogeneities in the distribution of cavities, the presence of a second population of secondary, smaller voids, etc. Significant contributions have already been made in this direction, but much remains to be done.

\subsubsection{FATIGUE CRACKING}

Generally, cracks leading to failure of structures originate from fatigue phenomena. Classically, the fatigue life is divided into "initiation" and "propagation" phases; depending on observation scales, the relative part of each phase is variable. In structural analyses, the crack initiation size is a few millimeters. For metallurgists, it may be of the order of microns. Some authors neglect the initiation phase [13]. Paris's law gives the crack propagation rate per cycle

$$
d a / d N=C\left(\Delta K_{1}\right)^{m}
$$

for long cracks in terms of the amplitude of stress-intensity factor variations $\Delta K_{\mathrm{l}}$. For these cracks, the defect will not grow if $\Delta K_{\mathrm{l}}<\Delta K_{\mathrm{th}}$; however, if the applied stress $S$ is sufficient high enough, $S>S_{f}$ (the fatigue limit), and cracks initiate and propagate until final rupture. In Kitagawa's diagram [12], based on Paris's law and the fatigue limit concept, the size of crack initiation corresponds to the region of LEFM applicability, which is of the order of $100 \mu$ or more for steels. Below this size, which 
corresponds to the domain of short cracks, studied by metallurgists at the scale of some microns. Different empirical formulae are proposed to correlate the crack growth rate with the local shear stress acting on the gliding plane of microcracks and/or plastic sliding shear along these planes. Nevertherless, the way to evaluate these quantities is questionable; these local quantities differs from macroscopic stress fields because the medium is no longer homogeneous at the microscopic scale. The scale to be considered is the grain size. There are stresses induced by incompatible plastic strain in grains. An attempt to evaluate the incompatible stresses is provided by Dang Van [7] for high cycle fatigue. This model is based on an elastic shakedown hypothesis at all scales.

\subsubsection{CONCLUSIONS}

The assumption of small-scale yielding allows us to apply LEFM to a variety of nonlinear situations, such as plasticity, damage, viscoplasticity, fatigue, etc. This is why LEFM is so popular in the engineering sciences. It explains the apparent paradoxical statement that a linear elastic theory can be used for describing complex situations involving nonlinearity. It also explains why a theory based on stress singularity does not contradict the small strain assumption of linear elasticity. LEFM is irrelevant in nonlinear phenomena in ductile rupture, with fully developed plasticity, with void growth, or in damage theory with localized phenomena. Today, such nonlinear phenomena can only be analyzed with some confidence by the so-called local approach to ductile rupture. However, there are still many engineering problems for which LEFM is still useful (for example, the fluidfilled crack problems, stress-corrosion cracking) or can be generalized to (cracks in electro-piezoelasticity, in coupled elasticity-electromagnetism, poroelasticity, etc.). Numerous unsolved problems in LEFM, particularly in the fields of inverse problems (crack detection, seismology, etc.) are also challenges.

\section{REFERENCES}

1. Achenbach, J. D., and Bazant, Z. (1972). Elastodynamic near tip stress and displacement fields for rapidly propagating cracks in orthotropic materials. J. Appl. Mech. 97: 183.

2. Bui, H. D. (1977). Stress and crack displacement intensity factors in elastodynamics. 4th Proc. Int. Conf. Fracture, vol. 3, Waterloo. 
3. Bui, H. D., Ehrlacher, A. (1981). Propagation of damage in elastic and plastic solids, in Advances in Fracture Mechanic, p. 533, vol. 3, Francois, D., et al., eds., Oxford-New York: Pergamon Press.

4. Bui, H. D. (1983). Associated path-independent J-integrals for separating mixed modes. J. Mech. Phys. Solids 31: 439.

5. Bui, H. D. (1993). Introduction aux problèmes inverses en mécanique des matériaux, Paris: Eyrolles. English translation Inverse Problems in the Mechanics of Materials: An Introduction, CRC Press, Boca Raton, 1994.

6. Cherepanov, G. P. (1968). Cracks in solids. Int. J. Solids Struct. 4: 811.

7. Dang Van, K., and Papadopoulos, T. Y. (1997). High cycle metal fatigue: From theory to applications.

8. Gologanu, M., Leblond, J.-B., Perrin, G., and Devaux, J. (1997). Recent extensions of Gurson's model for porous ductile metals, in Continuum Micromechanics, chapter 2, pp. 61-130, Suquet, P., ed., Springer-Verlag.

9. Gurson, A. L. (1977). Continuum theory of ductile rupture by void nucleation and growth: Part 1. Yield criteria and flow rules for porous ductile media. ASME J. Engng. Mat. Tech. 99: 2-15.

10. Hui, C. Y., and Riedel, H. (1981). The asymptotic stress and strain field near the tip of a growing crack under creep conditions. Int. J. Fract. 17: 409.

11. Kachanov, L. M. (1978). Crack under creep conditions. Izv. An SSR Mekhanika Tverdogo Tela 9(1): 57.

12. Kitagawa, H., and Takahashi, S. (1976). Applicability of fracture mechanics to very small cracks or cracks in the early stage. 2nd Int. Conf. on the Mechanics of Behavior of Materials. ICM2, Boston, Metal Parks, Ohio: American Metals Society.

13. Miller, K. (1997). The three thresholds for fatigue cracks propagation. Fatigue and Fracture Mechanics, pp. 267-286, vol. 27, Piascik, R. S., et al., eds.,

14. Rousselier, G. (1981). Finite deformation constitutive relations including ductile fracture damage. Proc. IUTAM Symposium, on ductile fracture and 3D constitutive equations, Dourdan. Nemat-Nasser, S. N., ed., North-Holland.

15. Rice, J. R. (1968). Mathematical analysis in the mechanics of fracture, in Fracture, p. 2 , Liebowitz, H., ed., New York: Academic Press.

16. Rice, J. R., and Tracey, D. M. (1969). On the ductile enlargement of voids in triaxial stress fields. J. Mech. Phys. Solids 17: 201-217.

17. Willis, J. (1997). Asymptotic analysis in fracture, Proceedings ICF9, vol. 4, 1849-1859, Karihaloo, B. K., et al., eds., Pergamon. 